\title{
Enhancing Human Perspectives in CPS Models: Application to Collaborative Problem-Solving in Translational Medicine
}

\author{
Keiichi SATO ${ }^{\text {ad, }, 1}$, Fei HU ${ }^{b}$, Santosh BASAPUR ${ }^{c}$, Raj C. SHAH ${ }^{c}$ and \\ Gunnar HARTVIGSEN ${ }^{\mathrm{d}}$ \\ allinois Institute of Technology \\ b Tongji University \\ ${ }^{\mathrm{c}}$ Rush University Medical Center \\ ${ }^{\mathrm{d}}$ UiT The Arctic University of Norway
}

\begin{abstract}
Cyber Physical Systems (CPS) have been developed for providing seamless, resilient and high-fidelity services in areas such as manufacturing, healthcare, agriculture, transportation and everyday living. Yet, conceptual and methodological frameworks of CPS that effectively incorporate human perspectives have not been well addressed in research and practice. The success of system design can only be achieved when all stakeholders can mentally and behaviorally position the system in the complex environments and situations by forming user knowledge and meanings through the system lifecycle. Such close human-system collaboration in CPS would enable the real-world system performance such as adaptivity, robustness and resilience in dynamic environments. The goal of this research is to enhance human perspectives by introducing a semiotic framework for representing different aspects of human and organizational meaning formation. It also intends to explore how the semiotic approach can be integrated with Human System Integration (HSI), CPS or Cyber Physical Human Systems (CPHS) methodologies to further enhance human perspectives in CPS lifecycles by transdisciplinary approaches. The paper then introduces an early stage of the project that applies the approach to represent a collaborative problem-solving infrastructure for clinical research process innovation in a multi-institutional translational medicine organization.
\end{abstract}

Keywords. Cyber Physical Systems, Human System Integration, transdisciplinary approach, semiotic approach. clinical studies, translational medicine

\section{Introduction}

The concept of Cyber Physical Systems (CPS) has been widely adopted in both research and practice of complex system development incorporating advanced technologies in areas such as computing, network, sensors, information and physical systems. CPS' goal is to implement seamless, effective, resilient and high-fidelity services for human and organizational activities such as industrial processes, healthcare, agriculture, transportation and everyday living. CPS integrate multiple subsystems across physical and cyber spaces such as numerous sensors, computing, data and knowledge bases, networks, process controllers and effectors. While such technologies can pervade our activity space and deliver services where and when we need them, the quality of these

${ }^{1}$ Corresponding Author, Mail: sato@id.iit.edu 
services and human-system interaction becomes critical for their effective development, deployment and usage. The National Institute of Standards and Technologies report on CPS framework points out that more research is required to better understand the relationship between a human operator's cognitive cycle and the CPS conceived, built, and operated by humans [1].

The goal of this paper is to introduce a roadmap and requirements for further development of transdisciplinary approaches that will enhance human perspective in CPS design. First, Chapter 1 will review scopes and issues of human modeling schemes in two existing approaches: Cyber Physical Human Systems (CPHS) that incorporate humans in CPS scope; Human System Integration (HSI) that addresses integration issues of systems, humans and organizations through the system lifecycle. Chapter 2 discusses qualitative representation of humans in the system used in Human Centered Design (HCD) and Semiotic Approach. Chapter 3 introduces the Semiotic Approach and Design Information Framework (DIF) as integration mechanisms between qualitative human concerns and technical system representation. Chapter 4 introduces an early stage of the project that applies the proposed approach to a design of a transdisciplinary collaborative problem-solving platform in Translational Medicine research. Chapter 5 summarizes issues and requirements found from the application case and introduces a roadmap for the further development of the application project.

\section{Modeling humans in CPS}

The original CPS concept primarily focused on technical systems. As the CPS technology became mature and the number of implementation cases increased in broad areas, awareness and needs for modeling humans in the CPS have been increasing. The concept of CPHS has been developed in order to address critical roles of humans in the system, human perspectives and performance [2] [3]. HSI and Socio-Technical System also have been addressing issues from the lenses of human factors and large-scale complex system design, operation, management and social impacts [4] [5].

\subsection{Cyber Physical Human Systems (CPHS)}

CPHS' primary approach for enhancing human concern in CPS is to integrate human subsystem models together with models of technical subsystems. One method is to apply technical modeling schemes such as Unified Modeling Language (UML) and its adaptation to systems engineering, System Modeling Language (SysML), to represent aspects of human subsystems such as activities and cognitive architecture. This allows the integration of human and technical subsystems into a unified representation of human and technical systems [7] [8]. This approach can be positioned in the scope and conceptual foundation of HSI research. The following section overviews HSI's conceptual and methodological research for enhancing human perspectives that is effectively extendable to the CPS context.

\subsection{Human System Integration}

HSI is defined by the US National Research Council: Human-system integration (HSI) is concerned with ensuring that the characteristics of people are considered throughout the system development process with regard to their selection and training, their 
participation in system operation, and their health and safety. It is also concerned with providing tools and methods meeting these same requirements to support the system development process [8]. Besides inheriting the human factors and systems engineering resources, HSI further extended the human factors scope to large-scale complex systems by including much wider concerns and scopes of humans in the system lifecycle such as decision making and organizations [9].

The HSI ontology domain diagram by Orellana and Madni in Figure 1 shows how HSI ontology defines a meta-model and constrains an extension profile to enable extensions of SysML and Model Based System Engineering (MBSE) for enhancing human considerations [5]. This enables better transdisciplinary understanding and communication of HSI concerns, and also identifies core building blocks for creating the ontology for human system interaction, interfaces, and integration [5].

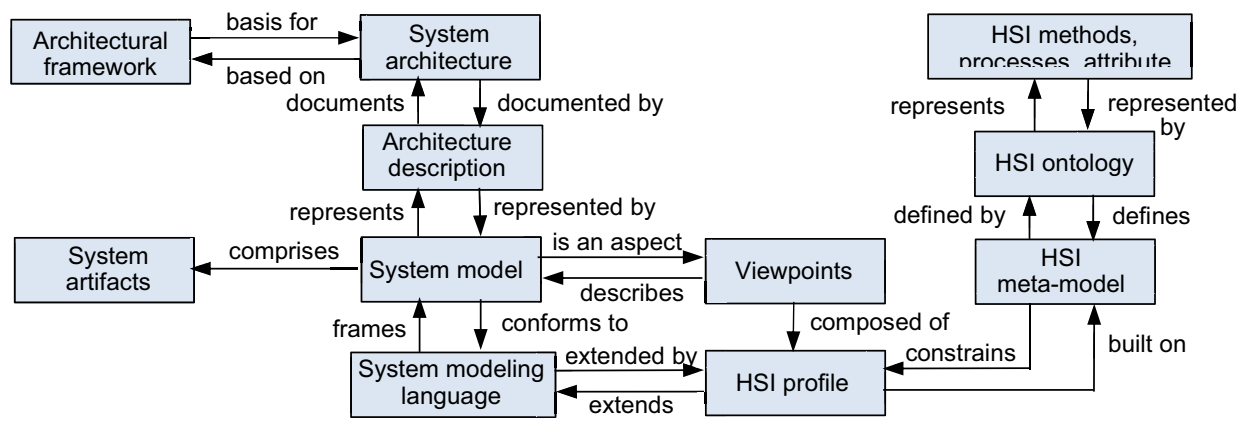

Figure 1. HIS Ontology Domain Diagram [5].

\section{Representing qualitative information in system design}

In the human engagement with systems, meaning generation, communication and interpretation are fundamental mechanisms to drive human action cycles. Meaning is fundamentally qualitative information. Here, the following sections explain how the Semiotic Approach can be used in the Human Centered Design context.

\subsection{Human Centered Design}

Human Centered Design (HCD) concept has been contributing to raise awareness and establish principles and guidelines concerning human viewpoints [6]. Yet, bridging the language of qualitative and conceptual information concerning humans (also referred as soft information) developed through field observation in the HCD approach and formal language of technical system discipline remains as a major challenge for pursuing holistic approaches to systems design. There are two fundamental questions, (1) how to combine qualitative information with formal representation, and (2) how to represent high-level concepts such as meanings and values.

\subsection{Semiotic frameworks for information systems design}

The approach based on the Semiotic Framework demonstrates a possible path to the issues represented by the two questions above. Many successful methods for quantifying "qualitative" information have been developed and applied in research and practice. In 
many cases, quantification enhances information; however, translation of qualitative information to a metric space often causes degradation and oversimplification. Therefore, subjects' information that is fundamentally qualitative in nature, such as meaning formation, transformation and interpretation processes and the nature of meaning itself can be better represented in logical form than quantitative form.

The Semiotic Approach in organization and information system design by Stamper and Liu focuses on sense making and meaningfulness to people [10] [11]. Stamper introduced the Semiotic Framework that reflects people's interaction with technical systems and organizational behavior. Three layers, physical, empiric and social layers, were added to the original framework of semiotics by Pierce composed of syntax, semantics and pragmatics as shown in Figure 2 [10]. The framework works as an interpretive mechanism for representing different levels and aspects of human and organizational meaning formation and behavioral reasoning. Stamper's framework became a foundation for organizational design and information system design by effectively addressing users views and needs from operational, organizational, social and cultural environments. With this framework, a comprehensive methodology of incorporating human perspectives into organization and information system design called MEASUR was developed [11]. The Semiotic Framework based methods have been used in the Semiotic Approach to Product Architecture design (SAPAD) for products and service systems design [12].

\begin{tabular}{|c|c|c|c|c|c|c|}
\hline \multirow{3}{*}{\multicolumn{3}{|c|}{$\begin{array}{l}\text { Human Information } \\
\text { Processing Functions }\end{array}$}} & \multirow[b]{3}{*}{ Meaning } & \multicolumn{2}{|c|}{ Social aspects } & $\begin{array}{l}\text { SOCIAL WORLD - belief, expectation, } \\
\text { commitment, contracts, law, culture }\end{array}$ \\
\hline & & & & Usage & $\begin{array}{l}\text { PRAGM } \\
\text { convers }\end{array}$ & $\begin{array}{l}\text { TICS - intention, communication, } \\
\text { tion, negotiation, etc. }\end{array}$ \\
\hline & & & & \multicolumn{3}{|c|}{$\begin{array}{l}\text { SEMANTICS - meanings, propositions, validity, truth, } \\
\text { signification, denotation, etc. }\end{array}$} \\
\hline IT Systems & & Structure & \multicolumn{4}{|c|}{$\begin{array}{l}\text { SYNTACTICS - formal structure, language, logic, data, } \\
\text { records, deduction, software, etc. }\end{array}$} \\
\hline & Access & \multicolumn{5}{|c|}{$\begin{array}{l}\text { EMPIRICS - pattern, variety, noise, entropy, channel capacity, } \\
\text { redundancy, efficiency, codes. etc. }\end{array}$} \\
\hline Media & \multicolumn{6}{|c|}{$\begin{array}{l}\text { PHYSICAL WORLD -signals, traces, physical distinction, hardware, } \\
\text { component density, speed, economics, etc. }\end{array}$} \\
\hline
\end{tabular}

Figure 2. Semiotic Framework by Stamper [10].

Norms are identified as forces that govern or influence human behavior and manifest as principles, guidelines, customs and conventions in social, cultural and organizational communities. They are implicitly referenced for forming interpretation, meaning, judgment and behavior. A norm addresses specific concerns, in other words, is formally or informally defined as permissible ranges in certain dimensions for meaning formation. At meta-levels, these dimensions can be overlaid on system models as constraints or specifications and integrated into systems development lifecycles.

\section{Bridging the Semiotic Approach with system modeling}

Disconnection between human concerns and technical system representation has been a major obstacle to ensuring the quality of new systems and system operations in organizations and our living environments. Human concerns are often elicited by field 
or user observation that result in textual and graphical documents of qualitative data. The compiled information from field and background studies are reviewed, analyzed, then insights are derived to identify requirements and generate specifications. Although some systematic methods and tools have been developed and used in practice, a lack of common ontology and language between qualitative research outcomes and formal documentation of technical system development impedes effective communication and collaboration between the two efforts.

\subsection{Design Information Framework (DIF) for bridging different views and representations}

The Design Information Framework (DIF) was introduced as a platform for bridging different representations by providing ontological structure for categorization and modularization of qualitative information. This mechanism can translate field notes, narrative documents, video or photographic documents through coding based on the ontological decomposition representing both human and technical perspectives [13] [14]. Figure 3 depicts the DIF platform and different formats of representation such as structured scenarios SysML models such as state models and sequence models [13]. The following chapter explains how DIF can bridge qualitative information of semiotic approaches to HSI ontology and system modeling languages to further enhance human perspectives.

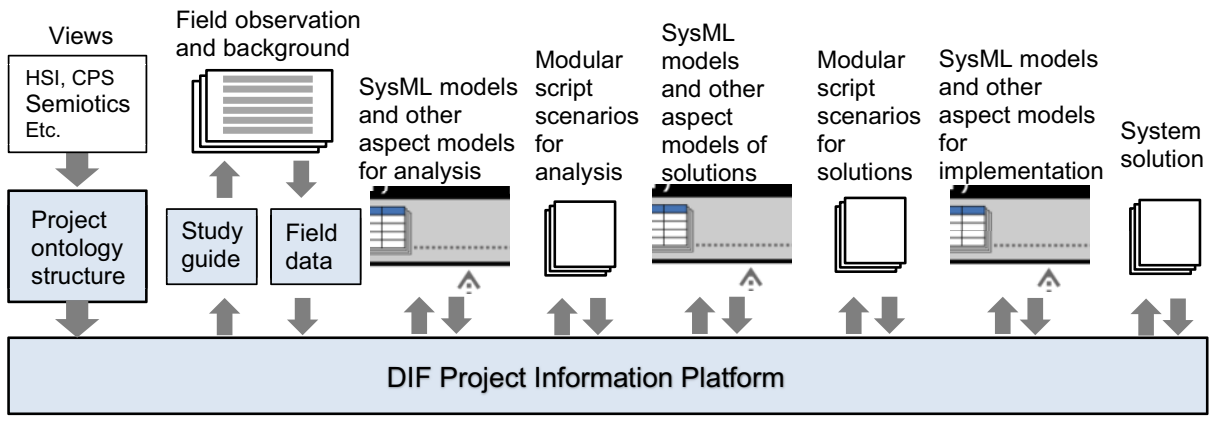

Figure 3. The process of generating scenarios and aspect models with DIF [13].

\subsection{Semiotic approaches to CPS development}

The original Semiotic Framework was introduced with its focus on understanding and analyzing organization and information systems. The upper three layers are allocated for understanding human information processing and the lower three layers are allocated for describing information systems. As Liu points out though, physical space and objects are also a critical part of the human work environment for information system usage [10]. Human cognition, knowledge, and learning are deeply embedded in the physical world through our activities. CPS performs sensing, decision making and social interactions that overlap with human activities. Therefore, all six levels of the semiotic framework should be applied to both human and technical systems. 


\section{Semiotic approach to ITM-TRIO program as service-oriented CPS}

The mission of the Institute for Translational Medicine (ITM) 2.0 [15] is to provide crossinstitutional infrastructure for promoting, educating, networking and supporting translational medicine/science. Its aims are: translational science workforce development; patient and community engagement in translational research (TR); equity across special and underserved population and lifespan in translational research; process innovation for quality and efficiency of multisite trials; and integration of advanced informatics. One of the key ways to achieve the aims is the TRIO Studio program. In this section, we highlight how the TRIO Studio is a service-oriented CPS and how the semiotic approach was utilized to achieve the work of the TRIO studio.

\subsection{TRIO Studio program as a service-oriented CPS}

The Trial Innovation Office (TRIO) architects the infrastructure for the crossinstitutional and transdisciplinary process innovation program that supports clinical research recruitment processes. The Studio's mission is to support clinical studies for enhancement of translational medicine. It has been providing collaborative and transdisciplinary problem-solving sessions (TRIO Studios) involving researchers, practitioners and administrators from diverse areas and roles of medicine, healthcare and sciences. The two-hour TRIO Studio hosts a topic presenter and participants consisting of researchers and administrators primarily from ITM member institutions. The presenter introduces issues of her/his clinical research problems, particularly in participant recruitment that are often bottlenecks of the project.

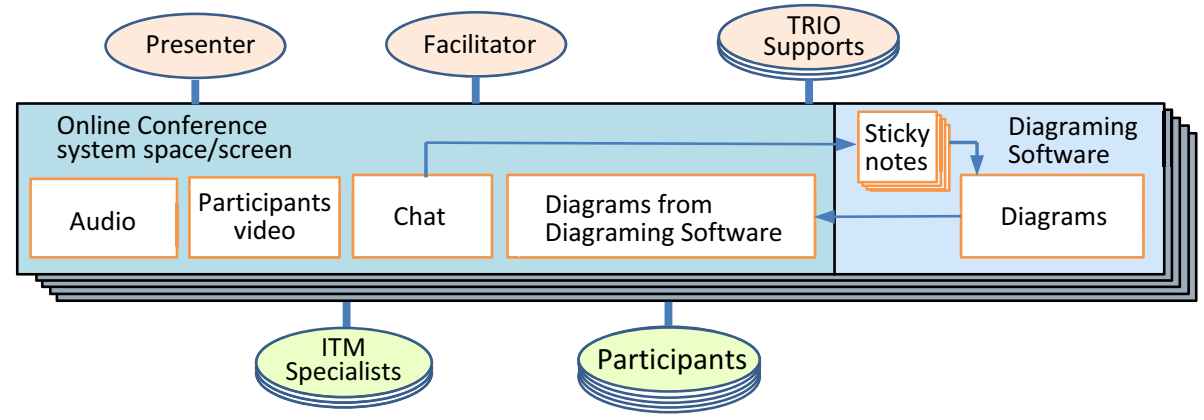

Figure 4. Configuration of online TRIO collaborative problem-solving sessions.

The roles involved in the Studio session are: 1) organizer who promotes the program, attracts presenters and participants and coordinates session plans and post session followup with presenters, 2) facilitator who navigates the discussion along the process of the chosen problem-solving approach, 3) session monitor who observes and analyses the session to maintain adherence to the chosen approach and suggest improvements, 4) TRIO staff for program support, 5) several ITM experts who are willing to share their experience and knowledge of problem-solving, 6) a topic presenter who presents his/her research project and problems and 7) participants who are interested in the topic and willing to contribute to the Studio. For productivity of the two-hour sessions, they are structured by one of two alternative approaches to problem-solving, Quality Science Approach (QSA) and Design Science Approach (DSA) for comparative studies between them (See [16] for detail). 
At its inception four years ago, the TRIO Studio was conducted in-person. However, COVID-19 restrictions required a transformation to an online delivery mode. The structured session format enabled relatively smooth transition from in-person to online modes. The online session interaction is supported by three channels, Zoom audio/video, Zoom chat function, and Mural visual collaboration software replacing whiteboards and sticky notes.

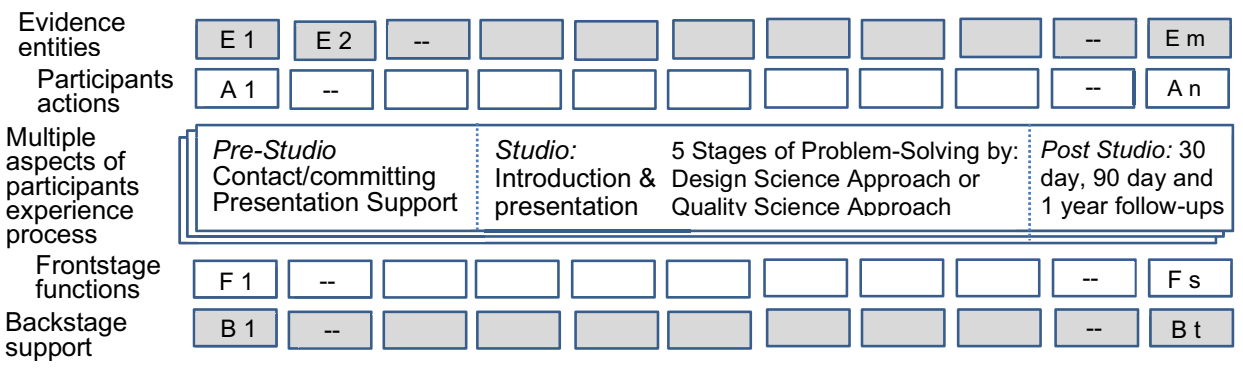

Figure 5. Service Blueprint View of TRIO Studio Process.

Figure 5 shows a service blueprint model of the general studio process with both human and technical system elements. In the model, the participants and the frontstage functions interact together and generate the system process and participants experience. Individual frontstage and backstage functions can be performed by human actor/s, system elements or both. A service action can be performed by any combination of human and system role allocations to frontstage and backstage based on the goal of the session design. The process is composed of three phases, preliminary, primary and follow-up phases. The primary phase is structured with five stages defined by either DSA or QSA.

\subsection{Semiotic approach for CPS analysis and envisioning of the TRIO Studio}

All the session records have been transcribed and coded with a coding scheme based on the research project ontology for further analysis. This coding scheme works as does DIF that enables further analysis by deriving formal representation with aspect models along with qualitative analysis on original documents.

The intent of applying the CPS framework to such case is to explore possibilities of the CPS framework as a platform for supporting human intensive system design and envisioning its transformation with advance technologies. The modality of the studio can range from fully in-person mode to fully virtual mode composed of all session functions supported or replaced by computerized tools and agents of CPS.

\begin{tabular}{|c|c|c|c|c|c|c|c|c|c|}
\hline time & phase & actor & act & object & function & objectiv & actor state & --- & $-\cdots$ \\
\hline 9:18 & $\begin{array}{l}\text { identifying } \\
\text { problems }\end{array}$ & A3 & propose & $\begin{array}{c}\text { alternative } \\
\text { view }\end{array}$ & $\begin{array}{c}\text { level } \\
\text { shift }\end{array}$ & $\begin{array}{l}\text { consistent } \\
\text { structure }\end{array}$ & $\begin{array}{l}\text { concerning } \\
\text { consistency }\end{array}$ & & \\
\hline $\begin{array}{l}\text { time } \\
\text { code }\end{array}$ & phase & actor & statement & $\begin{array}{c}\text { statement } \\
\text { type }\end{array}$ & $\begin{array}{c}\text { content } \\
\text { type }\end{array}$ & SF type & situatio & norm type & meaning \\
\hline 1236 & P3 & E & S-43 & B3 & process & L-5 & S7 & Role- & extra time \\
\hline & & & & & & & & &
\end{tabular}

Figure 6. Example of DIF coding. 
Some aspects of the human behavior and architectural models can be represented with SysML. For example, activity and sequence/interaction models of the Figure 5 process can be integrated with corresponding models of the technical subsystems as proposed by the CPHS frameworks. In order to enable transdisciplinary approaches to the complexity and heterogeneity of human systems, particularly to understand the governing forces of human-system behavior, the Semiotic Framework and other information categorization frameworks help researchers compose the ontological structure of the research information on the DIF platform. Use of the DIF platform with the embedded semiotic ontology enables quantitative and qualitative modeling for facilitating semiotic approaches in order to effectively explore human views of the system.

The upper row of Figure 6 shows an example of the general coding scheme of DIF documentation. Each row of the table accommodates an action description that is composed of the items specified by the scheme. Every project could have a different coding scheme based on the concern of the project. The example coding scheme works as: (at time: 9:18) (in phase of the process: identifying problems) (actor: A) (acted: propose) (on object: alternative view) (with function: level shift) (with objective: to consistent structure) (in actor state: concerning consistency). It is not necessary to fill all slots. The lower row of the figure shows an example of a data coding scheme that represent the information items relevant to the purpose and approach of the project. The example reads: (at time 1236) (in session phase 3) (expert E2) (make statement S-43) (about the problem B3) (in process) (referring Semiotic Frame: L-5 pragmatics) (S7: in general) (that relates to the norm type Role-5) (meaning: caught in detail). Currently the coding of approximately 20 sessions is in progress to track the patterns of the problemsolving process from problem identification, to solution emergence, to implementation.

\section{Discussion}

Reflective insights were gained through the session experiences and the review of recorded data. The following insight examples are more concerned with the general nature of the problem-solving session regardless of its mode.

- Visually shared memories such as diagrams, maps and lists for easy access are critical for summarizing and supplementing individual and group processes.

- In order to ensure flexible and cumulative discussion, the facilitator needs to ensure frequently reference to earlier discussion by using the earlier generated diagrams and effectively using the current diagrams in progress.

- The key role of experts is to provide solution architypes and relevant knowledge from their experience at appropriate times during the discussion.

A roadmap for possible transformation of the current TRIO platform to a CPHSbased platform can be set as follows:

Current project phase

- Identify information transformation patterns in the program process and nature of output concerning success, types, impact etc. from the session data analysis,

- Develop system models of the collaborative problem-solving session and platform that embodies the governing principles, strategy and approach of the next generation ITM program, Health Equity and Sociome. 
- Develop human-machine collaboration models of the TRIO program to envision future system development as a CPHS.

Next project cycle

- Implement an effective TRIO mission platform and collaborative problemsolving mechanisms for addressing health equity issues with social, cultural and ecological viewpoints (Sociome).

- Standardize the protocol and format for physical, online and hybrid modalities and achieve transportability, adaptivity and resilience by introducing modular system architecture with combinations of human actors and computing tools.

- Explore effective applications of Information and Communication Technology (ICT) to enhance TRIO session performance and experiment with these applications.

Possibilities beyond the scope of the current project goal

- Implement studio facilitation tools and knowledge bases to provide the best possible configuration of human actors and software tools in the Studio.

- Develop a self-learning, facilitation and simulation system for self-operating personal or small group studio sessions based on accumulated records of the previous studios and experts' knowledge in clinical research planning.

This roadmap for future system development of a collaborative problem-solving platform as a CPHS demonstrated the CPHS framework can work as a conceptual design tool for human intensive systems unlike most of current advanced technical systems where human roles are often limited.

Currently detailed study of the Studio session records is being conducted by coding and identifying patterns of collaborative problem-solving by a transdisciplinary community of the ITM organization. The result of this analysis will be combined with our insights and participants feedback and used to set the strategy for the next generation TRIO program as a service system. Integration of semiotic approaches with HSI, CPHS and service system frameworks on the DIF platform will be further explored to enhance CPHS design methodology.

\section{Conclusion}

This paper outlined the formulation of requirements for further development of transdisciplinary approaches for enhancing human perspectives in CPHS design. First, it reviewed scopes, capabilities and issues of human modeling schemes in existing approaches in HSI, Service System Design and CPHS. Second, it demonstrated a Semiotic Approach and DIF platform for structural representation of qualitative human information that can be integrated with technical system representation. This also works as a conceptual instrument for TRIO session record analysis in order to understand the collaborative problem-solving patterns. It will also be applied to identify issues and needs of future TRIO program planning in the ITM initiative. The next phase of the TRIO project study is to determine specific goals, requirements and conceptual specifications for future program development. A methodological architecture that closely links different frameworks and approaches including HIS, CPHS, semiotic approach, and DIF 
needs to be further developed to enhance scalability, accessibility and adaptivity for transdisciplinary approaches to system design.

\section{Acknowledgement}

This project was partly supported by the National Center for Advancing Translational Sciences (NCATS) of the National Institutes of Health (NIH) through Grant Number 5UL1TR002389-02 that funds the Institute for Translational Medicine (ITM). The content is solely the responsibility of the authors and does not necessarily represent the official views of the NIH. The TRIO Studio program is indebted to the presenters, participants as well as our TRIO staff.

\section{References}

[1] Cyber-Physical Systems Public Working Group, Framework for Cyber-Physical Systems: Volume 1, Overview, NIST Special Publication 1500-201, National Institute of Standards and Technology, 2017.

[2] S. K. Sowe, E. Simmon, I. Zettsu, F. deVaulx, and I. Bojanova, Cyber Physical-Human Systems: Putting People in the Loop, IEEE Computer Society IT Professional, Vol. 18-1, 2016, pp. 10-13.

[3] H. Stern and T. Becker, Development of a Model for the Integration of Human Factors in Cyber-physical Production Systems, Procedia Manufacturing, 2017, Vol. 9, pp. 151-158.

[4] M. Hause and M. Wilson, Integrated Human Factors Views in the Unified Architecture Framework, Annual INCOSE International Symposium (IS 2017), Vol. 27, Issue 1, July 2017, pp. 1054-1069.

[5] D.W. Orellana, A.M. Madni, Human System Integration Ontology: Enhancing Model Based Systems Engineering to Evaluate Human-System Performance, Procedia Computer Science, 2014, Vol. 28, pp. 19-25.

[6] M. E. Watson, C. F. Rusnock, J. M. Colombi, and M. E. Miller, Human-Centered Design Using System Modeling Language, Cognitive Engineering and Decision Making, Vol. 11-3, 2017, pp. 252-269.

[7] M. Krugh and L. Mears, A complementary Cyber-Human Systems framework for Industry 4.0 CyberPhysical Systems, Manufacturing Letters, Vol. 15- B, January 2018, pp. 89-92.

[8] R. W. Pew, A. S. Mavor (ed.), Human-System Integration in the System Development Process, The National Academies Press, Ashintong D.C., 2007.

[9] G.A. Klein, Naturalistic Decision Making, Human Factors: The Journal of the Human Factors and Ergonomics Society, 2008, Vol. 50-3, pp. 456-460.

[10] K. Liu, Semiotics in information systems engineering, Cambridge University Press, Cambridge, 2000.

[11] R. Stamper, K. Liu, M. Hafkamp and Y. Ades, Understanding the roles of signs and norms in organizations-a semiotic approach to information systems design, Behaviour \& Information Technology, 2000, Vol. 19-1, pp 15-27.

[12] F. Hu, J. Li, W. Wang and K. Sato, Meaningful Experience in Service Design for the Elderly: SAPAD Framework and its Case Study, In: International Conference on Engineering Design, July 2019, Delft, pp 3081-3090.

[13] Y-K. Lim and K. Sato, Describing multiple aspects of use situation: applications of Design Information Framework (DIF) to scenario development, Design Studies, 2006, Vol. 27, pp. 57-76.

[14] E-C. Jung, K. Sato, Y. Chen, X. He, T. MacTavish, D. Cracchiolo, DIF Knowledge Management System: Bridging Viewpoints for Interactive System Design, In: The Human-Computer Interaction International 2005 Conference, Las Vegas, July, 2005, pp. 423-437.

[15] Institute for Translational Medicine 2.0 (ITM 2.0), 2021, Trial Recruitment Innovation Office (TRIO), Accessed: 31.05.2021. [Online]. Available: https://chicagoitm.org/itm-trio/

[16] S. Basapur, R. Shah, K. Sato, S. Robinson, Infrastructuring Transdisciplinary Problem-Solving in Translational Medicine, Advances in Transdisciplinary Engineering, 2021, Vol. 15, in press. 